\title{
Fourier plane modeling of the jet in the galaxy M81
}

\author{
Arvind Ramessur ${ }^{1,2}$, Michael F. Bietenholz ${ }^{1,3}$, Lerothodi L. Leeuw ${ }^{2}$ \\ and Norbert Bartel ${ }^{3}$ \\ ${ }^{1}$ Hartebeesthoek Radio Astronomy Observatory, P.O. Box 443, \\ Krugersdorp, 1740, South Africa \\ ${ }^{2}$ College of Graduate Studies, University of South Africa, P.O. Box 392, \\ Unisa, 0003 Pretoria, South Africa \\ ${ }^{3}$ York University, Department of Physics and Astronomy, 4700 Keele Street, \\ Toronto, Ontario, M3J 1P3, Canada
}

\begin{abstract}
The nearby spiral galaxy M81 has a low-luminosity Active Galactic Nucleus in its center with a core and a one-sided curved jet, dubbed M81*, that is barely resolved with VLBI. To derive basic parameters such as the length of the jet, its orientation and curvature, the usual method of model-fitting with point sources and elliptical Gaussians may not always be the most appropriate one. We are developing Fourier-plane models for such sources, in particular an asymmetric triangle model to fit the extensive set of VLBI data of M81* in the $u-v$ plane. This method may have an advantage over conventional ones in extracting information close to the resolution limit to provide us with a more comprehensive picture of the structure and evolution of the jet. We report on preliminary results.
\end{abstract}

Keywords. galaxies: active, galaxies: individual (M81), galaxies: jets, instrumentation: high angular resolution, techniques: interferometric

\section{Introduction}

In the majority of Active Galactic Nuclei (AGN) that can be resolved with very long baseline interferometry (VLBI), the jet is brighter on one side and often shows curvature. However, there are many marginally resolved AGNs, such as the low-luminosity AGN (LLAGN) in M81, which we assume are similar to the well-resolved ones except that they are physically smaller. The difficulty in studying marginally resolved AGNs is in reliably extracting from the data the basic parameters of the jet such as its length, orientation and degree of bending.

In VLBI data analysis, model fitting is the choice for interpreting unresolved or barely resolved components in sources. However, the models mostly used by radio astronomers are point sources and elliptical Gaussians, which are symmetrical and have no curvature. For sources with a bent one-sided jet (Fig. 1$)$, such as the LLAGN $\left(\approx 10^{37.5} \mathrm{erg} \mathrm{s}^{-1}\right)$ in M81 (e.g., Bietenholz et al. 2000; 2004), henceforth M81*, more efficient models are needed that can parameterize asymmetry as well as curvature of the jet with a minimal number of parameters.

\section{Preliminary result and discussion}

We have determined the Fourier transform of a one-sided trianglar model (see, e.g., Bracewell 2001) and compare the real VLBI image of M81* to that of a convolved triangle and of a standard elliptical Gaussian model as shown in Fig. 2. Notice that the triangle model, unlike the Gaussian model reproduces the off-centre peak brightness point seen in the VLBI image. The triangle model is an asymmetric one, going linearly from $100 \%$ 


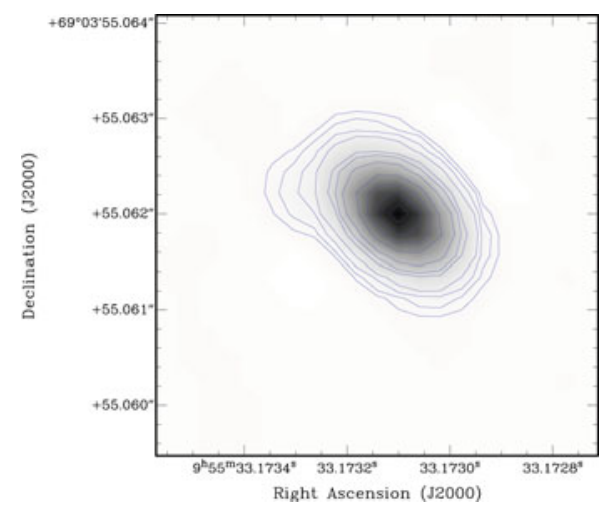

Figure 1. VLBI image at 8.4 $\mathrm{GHz}$ of $\mathrm{M} 81^{*}$, which is elongated and has a somewhat one-sided and bent jet. The contours are at $-2,2,5,10,20,30,40,50,60,70,80$ and $90 \%$ of the peak brightness of $110 \mathrm{mJy} \mathrm{beam}^{-1}$ and the FWHM resolution was $\sim 0.7$ mas. The observations are described in Bietenholz et al. (2004).
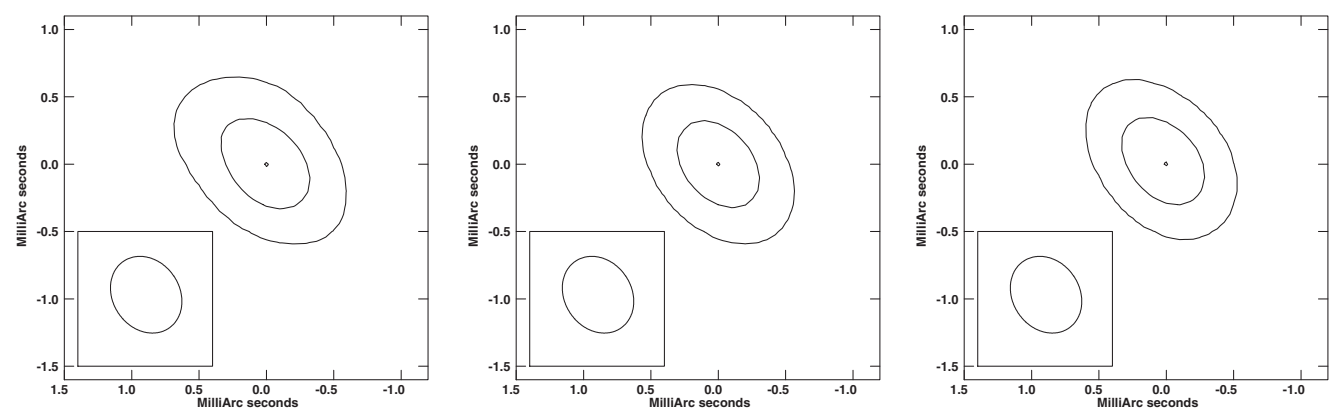

Figure 2. The VLBI image of M81* (left) compared to a fitted elliptical Gaussian model (center) and a triangle model (right) matched by eye, with the models convolved to a resolution comparable to that of the VLBI image. The contours are drawn at 10,50 and $90 \%$ of the peak brightness, and the FWHM resolution is indicated at the lower left.

brightness on one side to $0 \%$ on the other (i.e., a ramp function) over a length of 7.1 mas at P.A. of $45^{\circ}$ to match M81*.

Quantitative measurements of the physical parameters, in particular the length and bent of the jet require careful data modeling. We will expand our asymmetric triangle model to a two-sided asymmetrical model and a model with curved jets. This improved model will be fitted to the whole series of high resolution visibility data for M81* which will provide us with a robust estimation of the source geometry and its evolution with time. We will attempt to make a correlation analysis of the jet length, orientation and degree of bending with the flux densities and spectral indices. Lastly, we will also attempt to understand how the timescales of the jet variability relate to those seen in more luminous radio galaxies and quasars.

\section{Acknowledgements}

The financial assistance of the South African Square Kilometre Array (SA SKA) Project and Hartbeesthoek Radio Astronomy Observatory (HartRAO) towards this research is hereby acknowledged.

\section{References}

Bietenholz, M. F., Bartel, N., \& Rupen, M. P. 2000, ApJ, 532, 895

Bietenholz, M. F., Bartel, N., \& Rupen, M. P. 2004, ApJ, 615, 173

Bracewell, N. R. 2001, The Fourier Transform and Its Applications (3rd ed. New York: McGrawHill), pp. 61-65 